\title{
Diabetes mellitus and postoperative blood glucose value help predict posthepatectomy liver failure in patients with hepatocellular carcinoma
}

\author{
Jianchen $\mathrm{Luo}^{1} \wedge$, Liangliang $\mathrm{Xu}^{2} \wedge$, Lian $\mathrm{Li}^{1} \wedge$, Jingfu Zhang ${ }^{1} \wedge$, Ming $\mathrm{Zhang}^{2} \wedge$, Mingqing $\mathrm{Xu}^{2} \wedge$ \\ ${ }^{1}$ Department of General Surgery, West China Hospital, Sichuan University, Chengdu, China; ${ }^{2}$ Liver Surgery \& Liver Transplantation Center, West \\ China Hospital, Sichuan University, Chengdu, China \\ Contributions: (I) Conception and design: J Luo; (II) Administrative support: M Xu; (III) Provision of study materials or patients: J Luo, M Zhang; \\ (IV) Collection and assembly of data: J Luo, L Li; (V) Data analysis and interpretation: J Luo, L Xu; (VI) Manuscript writing: All authors; (VII) Final \\ approval of manuscript: All authors. \\ Correspondence to: Mingqing Xu, MD, PhD. Professor, Liver Surgery \& Liver Transplantation Center, West China Hospital, Sichuan University, No. \\ 37 Guo Xue Xiang, Chengdu 610041, China. Email: xumingqing0018@163.com.
}

Background: Many complications after hepatectomy can lead to perioperative death, among which posthepatectomy liver failure (PHLF) is the leading one. Existing studies suggest that one of the most important risk factors for PHLF is cirrhosis. Hepatitis B virus (HBV) infection is an important factor in the occurrence of cirrhosis, and the exact relationship between HBV infection and PHLF is not obvious. Diabetes mellitus and postoperative blood glucose are closely associated with liver regeneration, but its exact relationship with PHLF remains unclear.

Methods: We collected clinical indicators from 920 adult patients treated at the Liver Surgery and Transplantation Center of West China Hospital of Sichuan University from April 2009 and April 2019. We conducted a univariate analysis find out the risk factors of PHLF, follow by a multivariate analysis to ascertain the independent risk factors. Receiver operating characteristic (ROC) curves were plotted to evaluate the predictive efficiency of each risk factor.

Results: Following hepatectomy, 205 (22.2\%) of patients were diagnosed with PHLF. Several variables were confirmed to related with PHLF significantly: diabetes $[\mathrm{P}<0.01$, odds ratio $(\mathrm{OR})=10.845,95 \%$ confidence interval (CI): 5.450-21.579], $\mathrm{HBV}(\mathrm{P}<0.01$, OR $=0.345,95 \%$ CI: 0.187-0.635), blood glucose on the first postoperative day (post-BG1) $(\mathrm{P}=0.027$, OR $=1.059,95 \% \mathrm{CI}$ : 1.006-1.115), blood glucose on the third postoperative day (post-BG3) $(\mathrm{P}=0.021$, OR $=1.085$, 95\% CI: $1.012-1.162)$, blood glucose on the fifth postoperative day (post-BG5) ( $\mathrm{P}=0.014$, OR $=1.119,95 \% \mathrm{CI}: 1.023-1.225)$, postoperative total bilirubin (post-TB) $(\mathrm{P}<0.01, \mathrm{OR}=1.160,95 \% \mathrm{CI}: 1.133-1.187)$, and liver cirrhosis $(\mathrm{P}<0.01, \mathrm{OR}=0.982,95 \% \mathrm{CI}$ : 0.561-1.717) identified to be independent risk factors of PHLF.

Conclusions: Diabetes, HBV, post-BG1, post-BG3, and post-BG5 are related to the development of PHLF, and diabetes and post-BG can be used as predictors of the development of PHLF in patients with hepatocellular carcinoma (HCC).

Keywords: Diabetes; posthepatectomy liver failure (PHLF); hepatocellular carcinoma (HCC); receiver operating characteristic (ROC); area under the curve (AUC)

Submitted Jul 22, 2021. Accepted for publication Sep 02, 2021.

doi: 10.21037/jgo-21-491

View this article at: https://dx.doi.org/10.21037/jgo-21-491

\footnotetext{
^ ORCID: Jianchen Luo, 0000-0002-1352-4140; Liangliang Xu, 0000-0002-3900-9972; Lian Li, 0000-0002-8517-4661; Jingfu Zhang, 0000-0002-5069-3496; Ming Zhang, 0000-0002-8276-691X; Mingqing Xu, 0000-0002-8556-0802.
} 


\section{Introduction}

Hepatocellular carcinoma (HCC) is the third leading cause of cancer-related death worldwide and a common malignancy $(1,2)$. While hepatectomy remains one of the standard treatments for HCC (3), posthepatectomy liver failure (PHLF) remains a major problem for surgeons. Although the incidence of PHLF has decreased significantly after decades of patient selection and comprehensive preoperative evaluation, it remains the leading cause of mortality and morbidity after hepatectomy with $7 \%$ incidence $(4,5)$, and more comprehensive methods of predicting PHLF are required.

PHLF is mainly diagnosed by elevated postoperative international standardized rate (post-INR) and postoperative total bilirubin (post-TB) (4). Researchers have developed various parameters to predict of the development of PHLF, and liver cirrhosis is the foremost (6). However, whether underlying diseases such as diabetes can also be a risk factor for PHLF and abnormal biochemical indicators of patients before surgery can be identified, has not been fully clarified. Some studies have shown that prolonged hyperglycemia may further promote the development of cirrhosis by affecting liver regeneration, while preoperative or postoperative blood glucose levels have a direct effect on liver regeneration and the recovery of liver function (7). Hepatectomy in diabetic patients requires greater liver reserve function, patients infected with hepatitis $B$ virus (HBV) will have reduced liver function reserve due to cirrhosis caused by the virus, resulting in slow liver regeneration or even failure (8). However, it is unclear whether changes to postoperative blood glucose and its control in patients with and without diabetes can also be used as an indicator to predict the development of PHLF.

The purpose of this study was to evaluate risk factors for PHLF in a more comprehensive way. We evaluated the value of postoperative changes in blood glucose and postoperative blood glucose control in diabetic and nondiabetic patients as predictors of PHLF in HCC patients undergoing hepatectomy. We present the following article in accordance with the STARD reporting checklist (available at https://dx.doi.org/10.21037/jgo-21-491).

\section{Methods}

\section{Study design and patients}

This retrospective study included adult patients with HCC treated at the Liver Surgery \& Transplantation Center of West China Hospital of Sichuan University between April 2009 and April 2019, and the information of HCC patients undergoing hepatectomy was obtained from retrieval and extraction of its large database. Preoperative and postoperative clinical indicators were obtained by querying the data from the examination system. The study was conducted at the hospital between February 15, 2021, and May 30, 2021, and approved by the Ethics Committee of West China Hospital, Sichuan University (No. 2020/8), from which details can be obtained upon request. Individual consent for this retrospective analysis was waived. The study was conducted in accordance with the Declaration of Helsinki (as revised in 2013).

\section{Eligibility criteria}

The inclusion criteria were as follows: the patient's age was over 18 years; normal renal function and liver function reserves were present; hepatectomy was the initial treatment; and the statistical data of relevant indicators after surgery were relatively complete.

Exclusion criteria included: recurrent HCC, metastatic liver cancer caused by extrahepatic malignancy, portal vein and/or hepatic vein tumor thrombosis, or obstructive jaundice.

\section{Data source and pretreatment}

In this study, patients were required to have complete values of post-INR and post-TB on the fifth day after surgery, which were used to determine the diagnosis of PHLF and to draw baseline data. In addition, complete preoperative fasting blood-glucose (pre-FBG) values and postoperative blood glucose values on the first, third, and fifth days (post-BG1,3,5) were required. West China Hospital has strict preoperative examinations including routine blood, blood biochemistry, coagulation routine, blood type, and tumor markers. Perioperative management is rigorous with frequent monitoring of clinical indicators, while all blood samples are drawn before breakfast in the morning. These strict regulations made it possible to collect complete patient information and guarantee the validity and accuracy of the test results. Patients with incomplete clinical indicators were excluded, for example, those with missing postoperative clinical index data or with an unclear diagnosis of diabetes. The diagnosis of PHLF was made by two liver surgeons who were blinded to all patient information, including the final histopathologic diagnosis. 


\section{Diagnosis of HCC and diabetes}

To be diagnosed with HCC, a patient must have had two imaging tests that indicated the typical features, or one positive imaging result with a level of alpha-fetoprotein (AFP) greater than $400 \mathrm{ng} / \mathrm{mL}$, and the diagnosis was confirmed by pathology of the resected tissue after hepatectomy. Demographic, laboratory, and radiological data were collected for each patient in this study for further evaluation.

The diagnosis of diabetes was determined by reviewing the previous medical records of patients in part, and further confirmed by telephone follow-up. Patients previously diagnosed with diabetes but with abnormal recorded blood glucose levels, were asked to consult the hospital as soon as possible and provide timely feedback. In the absence of timely feedback, the diagnosis was defined as unclear.

\section{Definition of PHLF}

PHLF is defined as the impairment of liver function to maintain its synthesis, excretion, and detoxification that occurs after hepatectomy. According to the research of the International Study Group of Liver Surgery (ISGLS), PHLF is characterized by an increase in the INR on the fifth day after the operation or the operation, accompanied by hyperbilirubinemia $(4,9)$.

\section{Statistical analysis}

SPSS software (version 26.0) was used for statistical data analysis. Independent sample $t$-test was used to compare the continuous variables, and Fisher's exact test or chi-square test were used to compare the classification data. The risk factors for PHLF were evaluated by logistic regression analysis. Receiver operating characteristic (ROC) was used to analyze and evaluate the clinical value of pre-FBG and post-BG1, post-BG3, post-BG5. Sensitivity and specificity while area under the curve (AUC) and two-side $P$ values were calculated, and a $\mathrm{P}$ value was considered statistically significant when less than 0.05 .

\section{Results}

\section{Baseline data characteristics}

We extracted the information of 1,329 patients who underwent hepatectomy between April 2009 and April
2019 from the hospital database. By screening the integrity of clinical data and clarity of diagnosis, 284 patients were excluded, including 147 patients with missing data of postINR and post-TB $(\mathrm{n}=122)$, pre-FBG $(\mathrm{n}=9)$, and post-BG $(\mathrm{n}=103)$, and 37 patients because of an unclear diagnosis. A further 125 patients were excluded, including 43 who had undergone previous hepatectomy, 25 who received transhepatic arterial chemotherapy and embolization (TACE), 9 with portal vein or hepatic vein tumor thromboembolism, 37 whose pathologic reports indicated hepatic metastatic carcinoma, and 11 patients diagnosed with obstructive jaundice or other diseases of the blood system (Figure 1).

The mean age of the 920 patients was $50.92 \pm 11.88$ [22-82] years old, including 784 males (85.2\%) and 136 females (15.8\%). The baseline characteristics of the study population are shown in Table 1.

\section{Comparison of clinical indicators of diabetes patients and non-diabetes patients}

According to the follow-up of patients and the statistics of their medical records, 180 patients were diagnosed with diabetes. Table 2 shows a comparison of several potential clinical indicators in diabetes patients and non-diabetes patients, and reveals pre-FBG $(\mathrm{P}<0.05)$, post-BG1 $(\mathrm{P}<0.05)$, post-BG3 $(\mathrm{P}<0.05)$, post-BG5 $(\mathrm{P}<0.05)$, and post-TB $(\mathrm{P}<0.05)$ were found to be related to diabetes.

\section{Comparison of clinical parameters in patients with and without PHLF}

According to the diagnostic criteria, a total of 205 included patients were diagnosed with PHLF. Table 3 shows a comparison of several potential clinical indicators in PHLF patients and non-PHLF patients, and reveals pre-FBG $(\mathrm{P}<0.05)$, post-BG1 $(\mathrm{P}<0.05)$, post-BG3 $(\mathrm{P}<0.05)$, and postBG5 $(\mathrm{P}<0.05)$, were found to be related to PHLF.

\section{Analysis of risk factors for PHLF}

PHLF occurred in 205 HCC patients in this study, and we analyzed 32 potential variables to identify risk factors for PHLF in HCC patients, as shown in Table 4. Univariate analysis showed that diabetes, gender, HBV, pre-FBG, cystatin, post-BG, post-TB, main size, platelet (PLT), prothrombin time (PT), and liver cirrhosis were significantly correlated with the occurrence of PHLF. Multivariate 


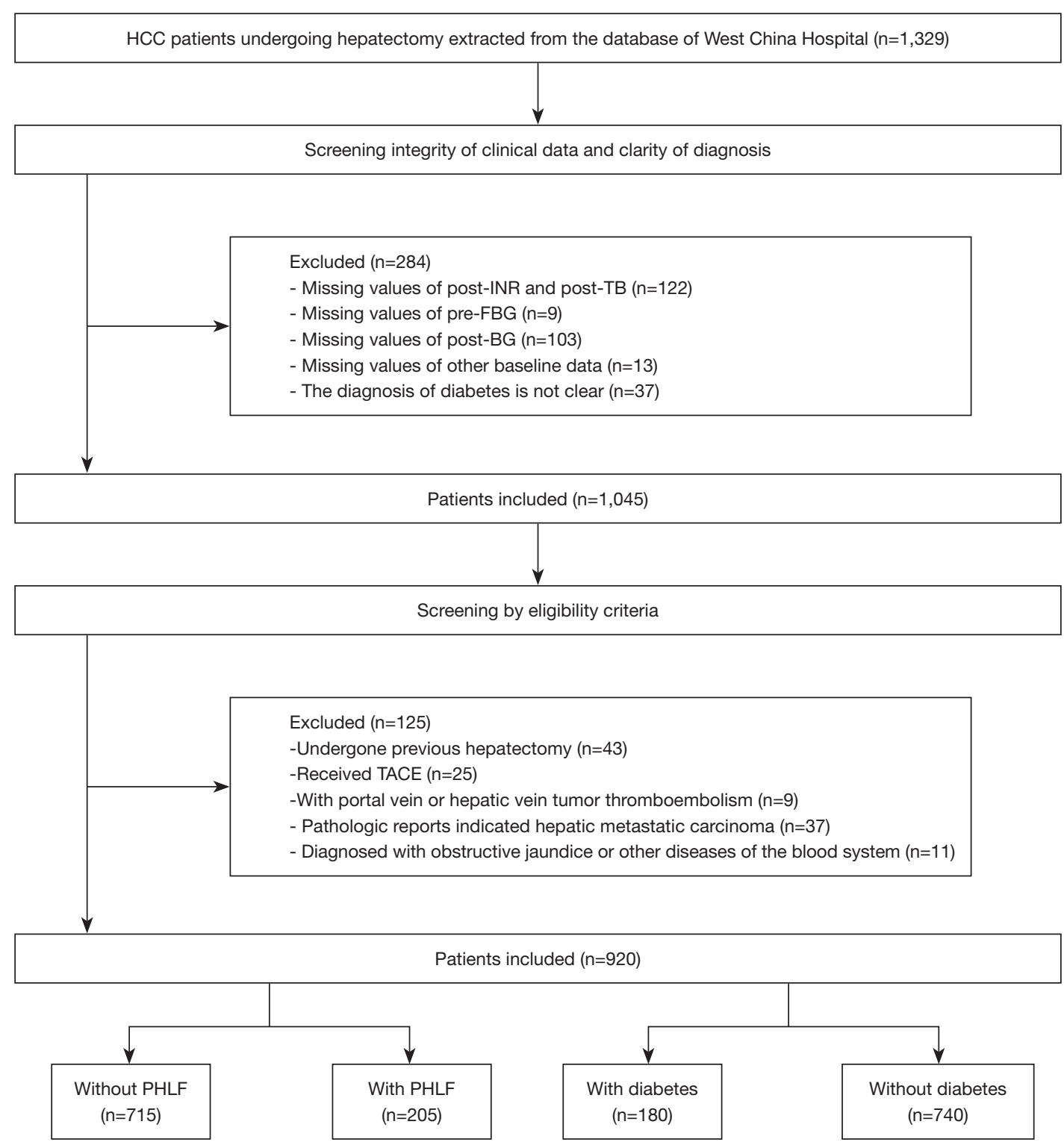

Figure 1 Screening of patients. HCC, hepatocellular carcinoma; post-INR, postoperative international normalized ratio; post-TB, postoperative total bilirubin; pre-FBG, preoperative fasting blood-glucose; post-BG, blood glucose on the postoperative day; TACE, transhepatic arterial chemotherapy and embolization; PHLF, posthepatectomy liver failure.

analysis demonstrated that diabetes $[\mathrm{P}<0.01$, odds ratio $(\mathrm{OR})=10.845,95 \%$ confidence interval (CI): 5.450-21.579], $\mathrm{HBV}(\mathrm{P}<0.01, \mathrm{OR}=0.345,95 \% \mathrm{CI}: 0.187-0.635)$, postBG1 ( $\mathrm{P}=0.027$, OR $=1.059,95 \%$ CI: $1.006-1.115)$, postBG3 $(\mathrm{P}=0.021$, OR $=1.085,95 \% \mathrm{CI}: 1.012-1.162)$, postBG5 ( $\mathrm{P}=0.014$, OR $=1.119$, 95\% CI: $1.023-1.225)$, postTB $(\mathrm{P}<0.01, \mathrm{OR}=1.160,95 \% \mathrm{CI}: 1.133-1.187)$, and liver cirrhosis $(\mathrm{P}<0.01, \mathrm{OR}=0.982,95 \%$ CI: $0.561-1.717)$ were independent risk factors for PHLF.

\section{Predictive effectiveness of post-BG1, post-BG3, and post-BG5 in development of PHLF}

ROC analysis was performed to assess the predictive effects of age, pre-FBG, and post-BG1, post-BG3, and post-BG5 of PHLF, as shown in Figure 2. The results showed that post-BG1 had an AUC of 0.607 ( $\mathrm{P}<0.001,95 \%$ CI: 0.4960.584 ), with a maximum joint sensitivity and specificity (sensitivity $=0.668$, specificity $=0.545)$ when the optimal cut- 
Table 1 Baseline data characteristics of the study population

\begin{tabular}{|c|c|}
\hline Variables & Value \\
\hline Age $(Y)$ & $50.92 \pm 11.88$ \\
\hline \multicolumn{2}{|l|}{ Gender } \\
\hline Male/female & $784 / 136$ \\
\hline \multicolumn{2}{|l|}{ Diabetes } \\
\hline Yes/no & $180 / 740$ \\
\hline \multicolumn{2}{|l|}{ PHLF } \\
\hline Yes/no & $205 / 715$ \\
\hline \multicolumn{2}{|l|}{ HBV } \\
\hline Yes/no & $795 / 125$ \\
\hline Pre-FBG (mmol/L) & $5.29 \pm 1.55$ \\
\hline Cystatin & $0.99 \pm 0.30$ \\
\hline Post-BG1 (mmol/L) & $7.61 \pm 3.18$ \\
\hline Post-BG3 (mmol/L) & $6.58 \pm 2.35$ \\
\hline Post-BG5 (mmol/L) & $6.12 \pm 1.77$ \\
\hline Post-TB ( $\mu \mathrm{mol} / \mathrm{L})$ & $26.50 \pm 16.81$ \\
\hline Post-INR & $1.24 \pm 0.76$ \\
\hline Main size $(\mathrm{cm})$ & $6.33 \pm 3.61$ \\
\hline Number of tumors & $1.10 \pm 0.61$ \\
\hline WBC (10느) & $5.74 \pm 3.17$ \\
\hline NEU $\left(10^{9} / \mathrm{L}\right)$ & $3.69 \pm 3.65$ \\
\hline LYM (10²/L) & $1.52 \pm 0.61$ \\
\hline MONO (10\% /L) & $0.36 \pm 0.17$ \\
\hline PLT (10º) & $142.63 \pm 71.72$ \\
\hline PT (s) & $12.30 \pm 4.29$ \\
\hline INR & $1.28 \pm 4.99$ \\
\hline APTT (s) & $28.08 \pm 4.09$ \\
\hline $\mathrm{Fib}$ & $2.82 \pm 1.05$ \\
\hline TB $(\mu \mathrm{mol} / \mathrm{L})$ & $15.04 \pm 6.56$ \\
\hline ALT (IU/L) & $56.57 \pm 67.72$ \\
\hline AST (IU/L) & $52.77 \pm 48.92$ \\
\hline TP (mmol/L) & $69.93 \pm 5.99$ \\
\hline ALB (g/L) & $41.18 \pm 4.57$ \\
\hline Creatinine $(\mu \mathrm{mol} / \mathrm{L})$ & $75.81 \pm 19.37$ \\
\hline \multicolumn{2}{|l|}{ MVI } \\
\hline Yes/no & $345 / 575$ \\
\hline
\end{tabular}

Table 1 (continued)
Table 1 (continued)

\begin{tabular}{lc}
\hline Variables & Value \\
\hline LCl & \\
Yes/no & $522 / 395$ \\
Satellite & \\
Yes/no & $126 / 794$ \\
Near invasion & \\
Yes/no & $39 / 881$
\end{tabular}

PHLF, posthepatectomy liver failure; HBV, hepatitis B virus; preFBG, preoperative fasting blood-glucose; post-BG1, blood glucose on the first postoperative day; post-BG3, blood glucose on the third postoperative day; post-BG5, blood glucose on the fifth postoperative day; post-TB, postoperative total bilirubin; post-INR, postoperative international normalized ratio; WBC, white blood cell; NEU, neutrophil; LYM, lymphocyte; MONO, monocyte; PLT, platelet; PT, prothrombin time; INR, international normalized ratio; APTT, activated partial thromboplastin time; TB, total bilirubin; ALT, alanine aminotransferase; AST, aspartate transaminase; TP, total protein; ALB, albumin; MVI, microvascular invasion; LCI, liver capsule invasion.

off value was set at 6.88; post-BG3 had an AUC of 0.591 $(\mathrm{P}<0.001,95 \%$ CI: $0.543-0.638)$, with a maximum joint sensitivity and specificity (sensitivity $=0.595$, specificity $=0.613$ ) when the optimal cut-off value was set at 6.03 ; and post-BG5 had an AUC of 0.604 ( $\mathrm{P}<0.001,95 \%$ CI: 0.558 $0.650)$, with a maximum joint sensitivity and specificity (sensitivity $=0.605$, specificity $=0.604$ ) when the optimal cut-off value was set at 5.86. No significant adverse events occurred in the analysis.

\section{Multivariate comparison of the effects of diabetes, $\mathrm{HBV}$, and post-BG on the occurrence of PHLF}

Multivariate analysis of variance (ANOVA) was performed to compare the effects of diabetes, $\mathrm{HBV}$, and post-BG on the occurrence of PHLF, and pairwise comparisons were performed to compare the subject effects (Figure 3). When diabetes and HBV acted together, the subject effect of both was significant (diabetes $\mathrm{F}=70.802, \mathrm{P}<0.001$; HBV $\mathrm{F}=15.903, \mathrm{P}<0.001)$, but the interaction effect was not significant (diabetes $+\mathrm{HBV} F=3.539, \mathrm{P}=0.06$ ). When diabetes and post-BG1, 3 , and 5 were combined, the subject effect of diabetes was significant $(\mathrm{F}=58.827, \mathrm{P}<0.001$; $\mathrm{F}=85.918, \mathrm{P}<0.001 ; \mathrm{F}=118.586, \mathrm{P}<0.001$ ), while the subject effect of post-BG was not significant (post-BG1 $\mathrm{F}=0.165$, 
Table 2 Comparison of clinical indicators in diabetes patients and non-diabetes patients

\begin{tabular}{lccc}
\hline Variables & Diabetes $(n=180)$ & Without diabetes $(n=740)$ & P value \\
\hline ALT & $60.06 \pm 78.79$ & $55.72 \pm 64.77$ & 0.10 \\
PLT & $127.61 \pm 64.22$ & $146.28 \pm 72.99$ & 0.65 \\
Cystatin & $1.04 \pm 0.24$ & $0.97 \pm 0.32$ & 0.43 \\
TB & $16.61 \pm 6.89$ & $14.66 \pm 6.43$ & 0.45 \\
Post-TB & $33.44 \pm 19.24$ & $24.81 \pm 15.72$ & $<0.05$ \\
Pre-FBG & $6.67 \pm 3.00$ & $4.94 \pm 0.76$ & $<0.05$ \\
Post-BG1 & $10.10 \pm 3.48$ & $7.00 \pm 2.78$ & $<0.05$ \\
Post-BG3 & $8.80 \pm 3.05$ & $6.03 \pm 1.76$ & $<0.05$ \\
Post-BG5 & $8.04 \pm 2.24$ & $5.64 \pm 1.25$ & $<0.05$ \\
\hline ALT,
\end{tabular}

ALT, alanine aminotransferase; PLT, platelet; TB, total bilirubin; post-TB, postoperative total bilirubin; pre-FBG, preoperative fasting bloodglucose; post-BG1, blood glucose on the first postoperative day; post-BG3, blood glucose on the third postoperative day; post-BG5, blood glucose on the fifth postoperative day.

Table 3 Comparison of clinical indicators in PHLF patients and non-PHLF patients

\begin{tabular}{lccc}
\hline Variables & PHLF $(\mathrm{n}=205)$ & Without PHLF $(\mathrm{n}=715)$ & $\mathrm{P}$ value \\
\hline Cystatin & $1.05 \pm 0.49$ & $0.97 \pm 0.22$ & 0.14 \\
Pre-FBG & $5.60 \pm 2.03$ & $5.19 \pm 1.36$ & $<0.05$ \\
Post-BG1 & $8.45 \pm 3.42$ & $7.37 \pm 3.07$ & $<0.05$ \\
Post-BG3 & $7.28 \pm 3.09$ & $6.38 \pm 2.04$ & $<0.05$ \\
Post-BG5 & $6.64 \pm 2.15$ & $5.97 \pm 1.62$ & $<0.05$ \\
\hline
\end{tabular}

PHLF, posthepatectomy liver failure; pre-FBG, preoperative fasting blood-glucose; post-BG1, blood glucose on the first postoperative day; post-BG3, blood glucose on the third postoperative day; post-BG5, blood glucose on the fifth postoperative day.

$\mathrm{P}=0.684$; post-BG3 $\mathrm{F}=0.616, \mathrm{P}=0.433$; post-BG5 $\mathrm{F}=8.233$, $\mathrm{P}=0.066$ ), and the interaction effects were not significant.

\section{Discussion}

PHLF remains a fearful complication after hepatectomy (10). While most studies on risk factors for PHFL have focused on the liver or spleen $(11,12)$, or patient related factors (13). Cirrhosis is considered to be a major risk factor for the development of PHLF (4). There are few examining perioperative patient management associated with the development of PHLF.

Most current studies have focused on the role of the liver itself in PHLF (14). Nishio et al. declared that liver stiffness (LS) could be a predictor of PHLF by analyzing the clinical indicators of $177 \mathrm{HCC}$ patients (15), and Berzigotti et al. reported that cirrhosis and portal hypertension could reflect LS, and the LS value could be an independent risk factor for PHLF in patients with HCC when greater than $15.7 \mathrm{kPa}$ (16). These results suggest LS is a simple, rapid, safe, and non-invasive method for the assessment of liver fibrosis and cirrhosis. In addition, recent studies have shown that spleen stiffness (SS) can also be used as an indicator of liver fibrosis or cirrhosis. Leung et al. reported that liver fibrosis and cirrhosis in HBV carriers could be distinguished by SS in different degrees (17), and SS was better than LS in predicting portal hypertension (18). Chen et al. suggested spleen thickness (ST) is a simple, inexpensive, and routinely available perioperative marker with good predictive value for postoperative prognosis of $\mathrm{HBV}$-associated $\mathrm{HCC}$ patients (19).

PHLF is comprised of a conundrum of symptoms and signs following major hepatic resection. Prompt identification of the pre-operative predictors of PHLF in 
Table 4 Analysis of risk factors for PHLF in patients with HCC

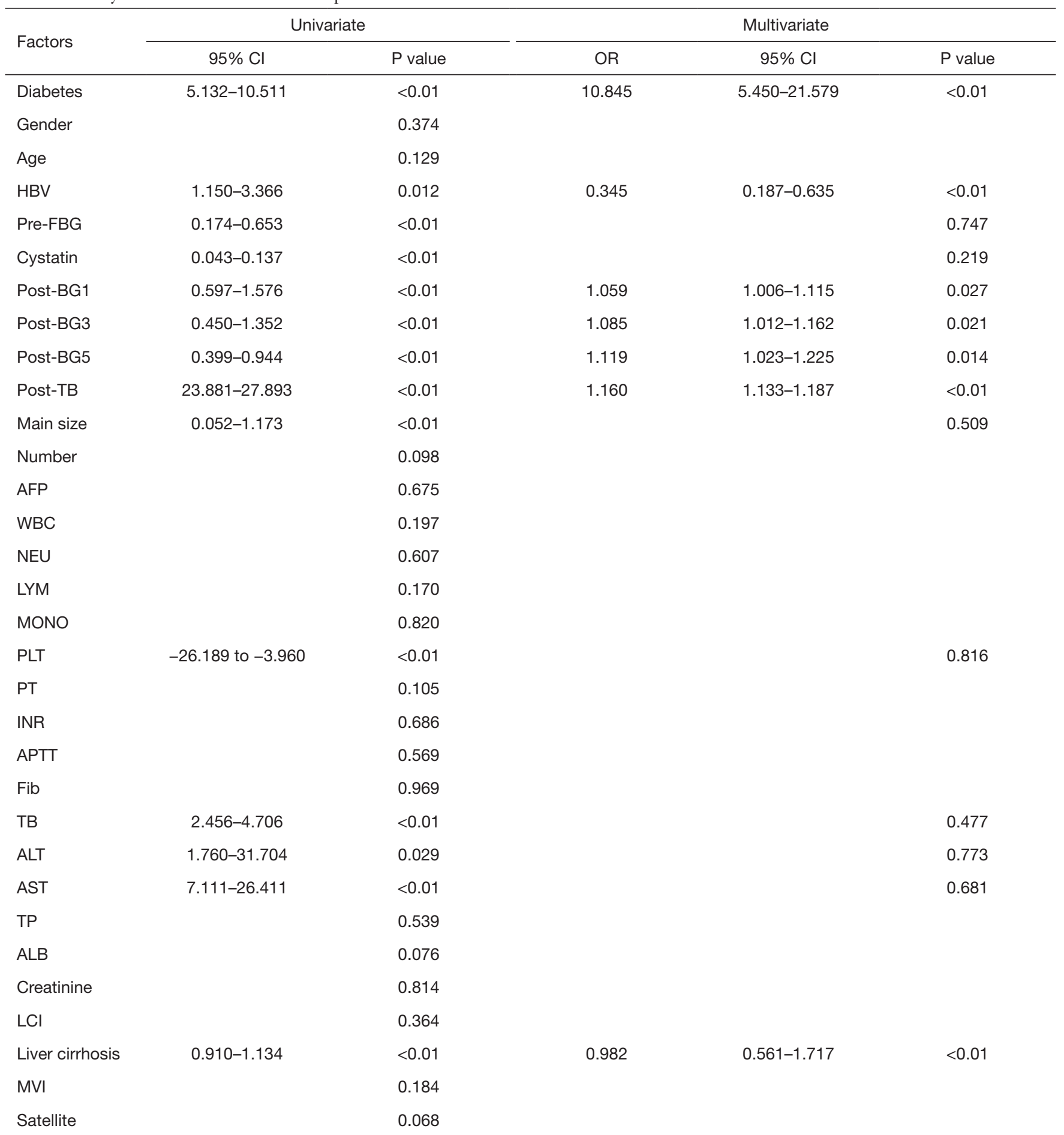

PHLF, posthepatectomy liver failure; HCC, hepatocellular carcinoma; $\mathrm{Cl}$, confidence interval; OR, odds ratio; HBV, hepatitis B virus; preFBG, preoperative fasting blood-glucose; post-BG1, blood glucose on the first postoperative day; post-BG3, blood glucose on the third postoperative day; post-BG5, blood glucose on the fifth postoperative day; post-TB, postoperative total bilirubin; AFP, alpha-fetoprotein; WBC, white blood cell; NEU, neutrophil; LYM, lymphocyte; MONO, monocyte; PLT, platelet; PT, prothrombin time; INR, international normalized ratio; APTT, activated partial thromboplastin time; TB, total bilirubin; ALT, alanine aminotransferase; AST, aspartate transaminase; TP, total protein; ALB, albumin; LCI, liver capsule invasion; MVI, microvascular invasion. 
the form of biochemical parameters and imaging features are of paramount importance for any hepatic surgeon and form the cornerstone of its management (20). However,

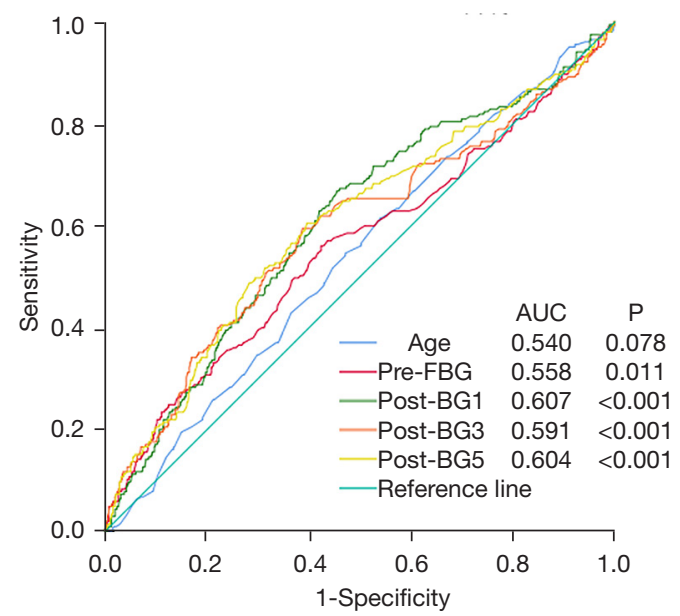

Figure 2 ROC analysis of pre-FBG, post-BG1, post-BG3, and post-BG5 for predicting development of PHLF. ROC, receiver operating characteristic; pre-FBG, preoperative fasting bloodglucose; post-BG1, blood glucose on the first postoperative day; post-BG3, blood glucose on the third postoperative day; post-BG5, blood glucose on the fifth postoperative day; PHLF, posthepatectomy liver failure; AUC, area under the curve. compared to the liver and spleen, few studies have been conducted on other clinical indicators of HCC patients which may also have a significant impact on postoperative recovery of patients. Liver regeneration is closely related to the occurrence of PHLF, and studies have shown that mesenchymal stem cell transplantation can be a potential treatment (21). Amini et al. proposed that the body mass index (BMI) of patients could have an impact on liver regeneration (22), and in a control study of 84 samples, Truant et al. found that obese patients had a relatively slow response to regeneration (23). Preoperative and postoperative blood glucose levels could also affect liver regeneration after hepatectomy (24).

Perioperative indicators that can potentially predict the occurrence of PHLF have become the focus of attention in several studies. There are also models designed to predict liver failure (25-28). Chin et al. claimed that the Model for End-stage Liver Disease (MELD) score, bilirubin, AFP, and PLT count showed significant predictive value for PHLF/insufficiency (PHLF/I) (all $\mathrm{P}<0.05)(29)$, while Xing et al. showed that preoperative TB, PLT, aspartate aminotransferase to PLT ratio index (APRI), and spleen volume/liver volume ratio $(\mathrm{SV} / \mathrm{LV})$ in patients with hepatectomy were independent risk factors for PHLF (30). Compared with this study, there are different opinions on PLT and preoperative bilirubin levels, which may be related
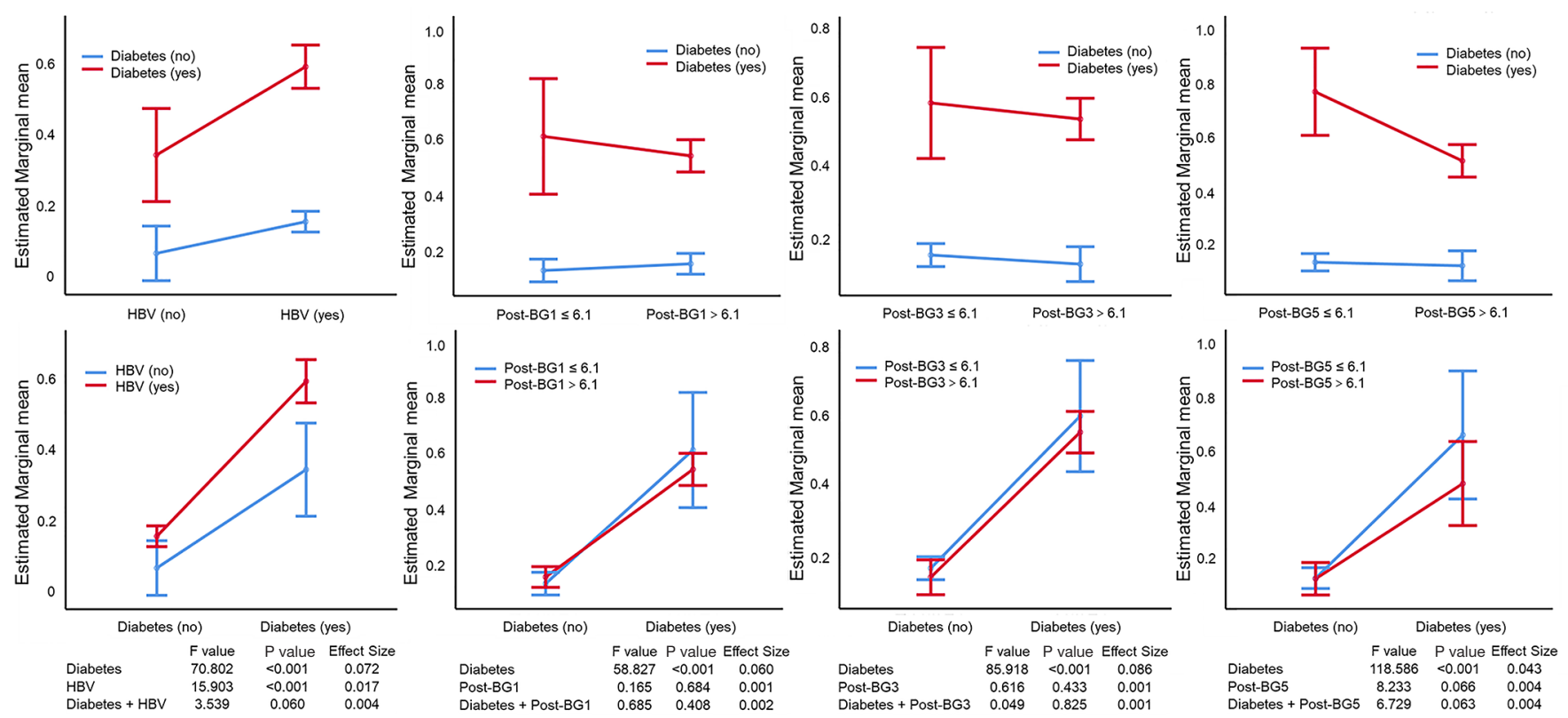

Figure 3 Multivariate comparison of the effects of diabetes, HBV, and post-BG on the occurrence of PHLF. HBV, hepatitis B virus; postBG1, blood glucose on the first postoperative day; post-BG3, blood glucose on the third postoperative day; post-BG5, blood glucose on the fifth postoperative day; PHLF, posthepatectomy liver failure. 
to the sample size.

In this study, we showed diabetes and postoperative blood glucose can be used as risk factors for PHLF, and have certain predictive significance for the its development in patients with HCC. The detrimental effects of hyperglycaemia include an increase in the oxidative stress (OS) response and an enhanced inflammatory response. Diabetes Mellitus compromises the ability of the liver to regenerate, and is associated with a poor prognosis after ischemia-reperfusion (I/R) injury (31). In addition, Wang et al. believed that diabetes drugs have toxicological effects on the liver, which may also be the reason why diabetic patients are more prone to develop PHLF (32).

The advantage of this study is that it further proves that diabetes is a risk factor for the occurrence of PHLF, and PHLF should be prevented in HCC patients with diabetes after hepatectomy. At the same time, this study further confirmed that postoperative blood glucose control had a certain predictive value on the occurrence of PHLF, and determined the relevant cut-off value.

Limitations to this study include the following: (I) all samples were from mainland China, and the applicability of the results to other regions remains to be verified, as the incidence of diabetes and PHLF is different in different regions (33-35); (II) our research mainly focused on liver cancer caused by HBV infection; therefore, this result may not be applicable to patients with other liver diseases, such as alcoholic liver disease, non-alcoholic fatty liver disease, and autoimmune hepatitis; (III) no further subgroup analysis was performed. Future work should evaluate the practical value of post-BG in predicting PHLF occurrence after hepatectomy, and involve more patients and research institutions.

According to the results of our study, in order to reduce the occurrence of PHLF, postoperative blood glucose control is very necessary, especially in patients with $\mathrm{HBV}$ infection or cirrhosis background. Most patients received hepatectomy will undergo fasting and abstentions after surgery, and the amount of glucose supplement should be carefully calculated and monitored during perioperative fluid rehydration.

\section{Acknowledgments}

Thanks to West China Hospital of Sichuan University and the Department of General Surgery for its support. Thanks for the technical support of Jinbang Kou.

Funding: This study was supported by the Key Technology
Research and Development Program of the Sichuan Province (2017FZ0082 and 2019YFS0208), the grants of National Natural Science Foundation of China (No. 71673193), and Chinese Foundation for Hepatitis Prevention and Control-Tian Qing Liver Disease Research Fund Subject (TQGB20190202).

\section{Footnote}

Reporting Checklist: The authors have completed the STARD reporting checklist. Available at https://dx.doi. org/10.21037/jgo-21-491

Data Sharing Statement: Available at https://dx.doi. org/10.21037/jgo-21-491

Conflicts of Interest: All authors have completed the ICMJE uniform disclosure form (available at https://dx.doi. org/10.21037/jgo-21-491). The authors have no conflicts of interest to declare.

Ethical Statement: The authors are accountable for all aspects of the work in ensuring that questions related to the accuracy or integrity of any part of the work are appropriately investigated and resolved. The study was conducted in accordance with the Declaration of Helsinki (as revised in 2013). The study was approved by the Research Ethics Committee of West China Hospital, Sichuan University, China (No. 2020/8). Individual consent for this retrospective analysis was waived.

Open Access Statement: This is an Open Access article distributed in accordance with the Creative Commons Attribution-NonCommercial-NoDerivs 4.0 International License (CC BY-NC-ND 4.0), which permits the noncommercial replication and distribution of the article with the strict proviso that no changes or edits are made and the original work is properly cited (including links to both the formal publication through the relevant DOI and the license). See: https://creativecommons.org/licenses/by-nc-nd/4.0/.

\section{References}

1. Torre LA, Bray F, Siegel RL, et al. Global cancer statistics, 2012. CA Cancer J Clin 2015;65:87-108.

2. Chen $W$, Zheng R, Baade PD, et al. Cancer statistics in China, 2015. CA Cancer J Clin 2016;66:115-32.

3. Jaeck D, Bachellier P, Oussoultzoglou E, et al. Surgical 
resection of hepatocellular carcinoma. Post-operative outcome and long-term results in Europe: an overview. Liver Transpl 2004;10:S58-63.

4. Rahbari NN, Garden OJ, Padbury R, et al. Posthepatectomy liver failure: a definition and grading by the International Study Group of Liver Surgery (ISGLS). Surgery 2011;149:713-24.

5. Mullen JT, Ribero D, Reddy SK, et al. Hepatic insufficiency and mortality in 1,059 noncirrhotic patients undergoing major hepatectomy. J Am Coll Surg 2007;204:854-62; discussion 862-4.

6. Poon RT, Fan ST. Hepatectomy for hepatocellular carcinoma: patient selection and postoperative outcome. Liver Transpl 2004;10:S39-45.

7. Singh KK, Panda SK, Shalimar, et al. Patients with diabetes mellitus are prone to develop severe hepatitis and liver failure due to hepatitis virus infection. J Clin Exp Hepatol 2013;3:275-80.

8. Nitta N, Yamamoto Y, Sugiura T, et al. Differences in the safety line of the future liver remnant plasma clearance rate of indocyanine green necessary to prevent posthepatectomy liver failure associated with underlying diseases. Surg Today 2021. [Epub ahead of print]. doi: 10.1007/s00595-021-02310-9.

9. Balzan S, Belghiti J, Farges O, et al. The "50-50 criteria" on postoperative day 5: an accurate predictor of liver failure and death after hepatectomy. Ann Surg 2005;242:824-8, discussion 828-9.

10. Schreckenbach T, Liese J, Bechstein WO, et al. Posthepatectomy liver failure. Dig Surg 2012;29:79-85.

11. Maithel SK, Kneuertz PJ, Kooby DA, et al. Importance of low preoperative platelet count in selecting patients for resection of hepatocellular carcinoma: a multiinstitutional analysis. J Am Coll Surg 2011;212:638-48; discussion 648-50.

12. Narita M, Oussoultzoglou E, Bachellier P, et al. Posthepatectomy liver failure in patients with colorectal liver metastases. Surg Today 2015;45:1218-26.

13. Ocak İ, Topaloğlu S, Acarli K. Posthepatectomy liver failure Turk J Med Sci 2020;50:1491-503.

14. Liaw YF, Chu CM. Hepatitis B virus infection. Lancet 2009;373:582-92.

15. Nishio T, Taura K, Koyama Y, et al. Prediction of posthepatectomy liver failure based on liver stiffness measurement in patients with hepatocellular carcinoma. Surgery 2016;159:399-408.

16. Berzigotti A, Reig M, Abraldes JG, et al. Value of transient elastography measured with fibroscan in predicting the outcome of hepatic resection for hepatocellular carcinoma. Ann Surg 2015;261:e105.

17. Leung VY, Shen J, Wong VW, et al. Quantitative elastography of liver fibrosis and spleen stiffness in chronic hepatitis B carriers: comparison of shear-wave elastography and transient elastography with liver biopsy correlation. Radiology 2013;269:910-8.

18. Sharma P, Kirnake V, Tyagi P, et al. Spleen stiffness in patients with cirrhosis in predicting esophageal varices. Am J Gastroenterol 2013;108:1101-7.

19. Chen X, Zou H, Xiong L, et al. Predictive power of splenic thickness for post-hepatectomy liver failure in $\mathrm{HBV}$-associated hepatocellular carcinoma patients. World J Surg Oncol 2017;15:216.

20. Ray S, Mehta NN, Golhar A, et al. Post hepatectomy liver failure - A comprehensive review of current concepts and controversies. Ann Med Surg (Lond) 2018;34:4-10.

21. Ding HR, Wang JL, Tang ZT, et al. Mesenchymal stem cells improve glycometabolism and liver regeneration in the treatment of post-hepatectomy liver failure. Front Physiol 2019;10:412.

22. Amini N, Margonis GA, Buttner S, et al. Liver regeneration after major liver hepatectomy: Impact of body mass index. Surgery 2016;160:81-91.

23. Truant $S$, Bouras AF, Petrovai G, et al. Volumetric gain of the liver after major hepatectomy in obese patients: a casematched study in 84 patients. Ann Surg 2013;258:696-702; discussion 702-4.

24. Margonis GA, Amini N, Buettner S, et al. Impact of perioperative phosphorus and glucose levels on liver regeneration and long-term outcomes after major liver resection. J Gastrointest Surg 2016;20:1305-16.

25. Deng $M, N g$ SWY, Cheung ST, et al. Clinical application of Albumin-Bilirubin (ALBI) score: the current status. Surgeon 2020;18:178-86.

26. Kamath PS, Kim WR; Advanced Liver Disease Study Group. The model for end-stage liver disease (MELD). Hepatology 2007;45:797-805.

27. Peng Y, Qi X, Guo X. Child-Pugh versus MELD score for the assessment of prognosis in liver cirrhosis: a systematic review and meta-analysis of observational studies. Medicine (Baltimore) 2016;95:e2877.

28. Kokudo T, Hasegawa K, Shirata C, et al. Assessment of preoperative liver function for surgical decision making in patients with hepatocellular carcinoma. Liver Cancer 2019;8:447-56.

29. Chin KM, Allen JC, Teo JY, et al. Predictors of posthepatectomy liver failure in patients undergoing extensive 
liver resections for hepatocellular carcinoma. Ann Hepatobiliary Pancreat Surg 2018;22:185-96.

30. Xing Y, Liu ZR, Yu W, et al. Risk factors for posthepatectomy liver failure in 80 patients. World J Clin Cases 2021;9:1793-802.

31. Mendes-Braz M, Martins JO. Diabetes mellitus and liver surgery: the effect of diabetes on oxidative stress and inflammation. Mediators Inflamm 2018;2018:2456579.

32. Wang T, Shankar K, Ronis MJ, et al. Mechanisms and outcomes of drug- and toxicant-induced liver toxicity in diabetes. Crit Rev Toxicol 2007;37:413-59.

33. Shehta A, Farouk A, Fouad A, et al. Post-hepatectomy

Cite this article as: Luo J, Xu L, Li L, Zhang J, Zhang M, Xu M. Diabetes mellitus and postoperative blood glucose value help predict posthepatectomy liver failure in patients with hepatocellular carcinoma. J Gastrointest Oncol 2021;12(5):23772387. doi: 10.21037/jgo-21-491 liver failure after hepatic resection for hepatocellular carcinoma: a single center experience. Langenbecks Arch Surg 2021;406:87-98.

34. Unnikrishnan R, Anjana RM, Mohan V. Diabetes mellitus and its complications in India. Nat Rev Endocrinol 2016;12:357-70.

35. Zheng Y, Ley SH, Hu FB. Global aetiology and epidemiology of type 2 diabetes mellitus and its complications. Nat Rev Endocrinol 2018;14:88-98.

(English Language Editor: B. Draper) 